\title{
Metastatic Model of Cerebellar Medulloblastoma Cells to Peritoneal Cavity: Exploration of Circulating Tumor Cells
}

\author{
P. Mehdipour ${ }^{1, *}$, F. Javan ${ }^{1}$, M. Faghih Jouibari ${ }^{2}$ and M. Khaleghi ${ }^{2}$ \\ ${ }^{1}$ Department of Medical Genetics, School of Medicine, Tehran University of Medical Sciences, Tehran-14176- \\ 1315, Iran \\ ${ }^{2}$ Department of Neurosurgery, Shariati Hospital, Tehran University of Medical Sciences, Iran
}

\begin{abstract}
Background: Circulating Tumor Cells (CTCs) are the reliable key for an early detection. The cellbased/classified/personalized diagnostic approaches are unavailable. Therefore, it was aimed to explore the expression behavior of tumor ( $\mathrm{T}$ ) cells in brain, peritoneal cavity (PC) and genomic level to deliver the hypothetical model through the metastatic events.

Patients and Methods: The focal assay included protein expression (PE) by immunofluorescence in T-cells of cerebellarmeduloblastoma (CM), PC, and CTCs in a metastatic patient. The CCL2, VEGF, EGF, CD133/Cyclin E/ P21/Neuronal marker (NM), and CD45 were explored.

Result: Frequency of T-cells lacking PE and the Ratio of T/CTCs in different sections of CM- tumor cells in brain and the metastatic PC revealed the diverse expression and co-expression of the involved proteins. The poor prognosis is offered upon the value of PE at T/CTCs ratio. High PE and harmonic co-expression played the influential role in the metastatic process and manner of evolution.

Conclusions: Single cell- based analysis of expression and co-expression is the directive channel to unmask the heterogeneity through the metastatic process at genomic and somatic levels for providing the metastatic model. Present findings deliver the somatic/genomic ratio-based prognosis for further clinical managements.
\end{abstract}

Keywords: Circulating tumor cells (CTCs), cerebellar medulloblastoma, peritoneal cavity, protein expression, Somatic/genomic heterogeneity, Metastatic model (MM).

\section{INTRODUCTION}

Medulloblastoma (MB) most frequently occurs among the infants and the middle aged individuals. MB is involved in the original cell population for cerebellum development [1]. Metastatic event from brain to another destination is rather rare. However, there are limited publications available on the extracranial metastasis of glioblastoma multiform (GBM) [2]. It was reported that the circulating tumor cells (CTCs) in GBM are characterized with an invasive mesenchymal nature into the blood stream [3]. The role of CTCs in different cancers has been highlighted, but there are limited available reports on the metastatic glioma as an invasive neoplasm $[4,5]$. However, there is no available report on cerebellar Medulloblastoma (CM).

Macrophage chemoattractant $\mathrm{C}-\mathrm{C}$ chemokine ligand 2 (CCL2) is essential for inflammation of brain, cell migration, and is capable to activate different cells. Furthermore, CCL2 has a directive role in transferring process over the blood brain barrier [6]. Vascular Endothelial Growth Factor (VEGF) family has a remarkable capability of vascularization [7]. So,

*Address correspondence to this author at the Department of Medical Genetics, School of Medicine, Tehran University of Medical Sciences, Tehran14176-1315, Iran; Tel: +9821-888302820; E-mail: mehdipor@tums.ac.ir angiogenesis has the functional impact on tumor development and progression. Epidermal growth factor (EGF) plays a key role in differentiation, proliferation, tumorigenic processes and apoptosis. Expression of this family member is found to be diverse in neoplasms and normal tissues. The over expression of EGFRs are reported in the epithelial tumors [8]. Due to the crucial impact of metastasis on the life style of the patients with brain neoplasms, tracing of CTCs could be considered as an early detecting biomarker for these patients. However, no report on tracing the CTCs based on the combined profile including CCL2/VEGF/EGF was available in patients with Medulloblastoma. Therefore, the main aim of present work was to explore this triple profile in different tissues of a patient with an extracranial $\mathrm{CM}$-cell migration to $P C$.

\section{MATERIALS AND METHODS}

\section{Patients}

A 39 years old female patient has gone through the brain surgery with histopathological diagnosis of $\mathrm{CM}$ and micro-vascular proliferation/ grade IV/IV. She was positive for D1853N polymorphism in ATM gene [9]. Regarding family history, her brother was affected with bone cancer at the age of 25- year. 
The tumor $(\mathrm{T})$ and peripheral blood (PB) samples were referred from Sharyati hospital. Enumeration of total T-cells in CM, PC and CTCs were 18659, 10794 and 118 respectively. The ratio of T/CTCs was considered for four and three different tumor sections of CM and PC respectively (Figure S1). The analytical strategy was based on the comparison of the cell frequency with different degree of intensity including 1 : low, 2: intermediate and 3: high and lack (zero) of proteins expression (PE) for CCL2: C, EGF: E and VEGF: $V$. The manual cell enumeration facilitated the observation of heterogenic pattern of $\mathrm{PE}$ and coexpression, reflecting the quantitative and qualitative status in T-cells, CTCs, different clone of cells and vasculature system.

\section{Samples and Materials}

Tumor samples and four $\mathrm{ml}$ of heparinized peripheral blood (PB) were used for cell extraction. After the washing process with $1 \mathrm{x}$ phosphate buffer solution, the cells were stained with anti- CCL2 (eBioscience), VEGF (Genature, Belgium) and EGF (Novous Biologicals, U.S.A.). CD133 (Biolegend, U.S.A), and Cyclin E (Zymed, USA) were also examined. Then, the cells were incubated at $4{ }^{\circ} \mathrm{C}$ and stained with second layer antibodies comprising of FITC, R-Pe and Pe-Cy5.The nuclear dye (DAPI) is added and the mode of PE was observed by the LEICA, DM RXA2-fluorescence microscope (Germany). Specific markers including, leukocyte common antigen (LCA cocktail (CD45) (Bio care, The Netherlands) and neuronal marker (NM) (ScyTek, The Netherlands) were also used for confirming the tissue origin of CTCs. Definition of the CTCs was confirmed

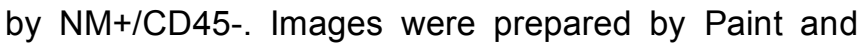
the resolution was optimized by Adobe illustrator 2017 .

Based on the personalized perception of present study, estimation of Ratio between the T-cells in tumors, CTCs and vascular system was performed.

The project was approved by the deputy of Research, Tehran University of Medical Sciences (32208-30-04-95). The written consent declaration was obtained from the patient.

\section{RESULTS}

Exploration of T/CTCs-ratio was based on distribution of: 1) diverse intensity-based $P E$ in $C, V$, and $E$ in the samples of each CM- and PC-tumors; and 2) cells lacking expression of these proteins. The harmonic manner of ratio for each protein is presented with quadrant boxes (Figure S1). The mode of PE and co-expression is provided for: Profile of CCL2, VEGF and EGF (Figure 1); the CM migrated cells to the section 1 of PC (Figure 2); the migrated cells from CM to the vasculature of section 2 in PC (Figure 3); and for the migrated $\mathrm{CM}$ cells to the section 3 of PC (Figure 4). PE of CD133, Cyclin E and P21 in the CM cells of the brain and peritoneal cavity is presented (Figure 5). Regarding the origin of T-cells, status of PE for NM, CD133 and VEGF in the section 1 of migrated CM cells to the vascular region of peritoneal cavity is provided (Figure 6). Mode of PE and co-expression of CCL2, VEGF and EGF in the CTCs; and profile of CD133, Cyclin E, and P21 in CTCs from CM to PC are a

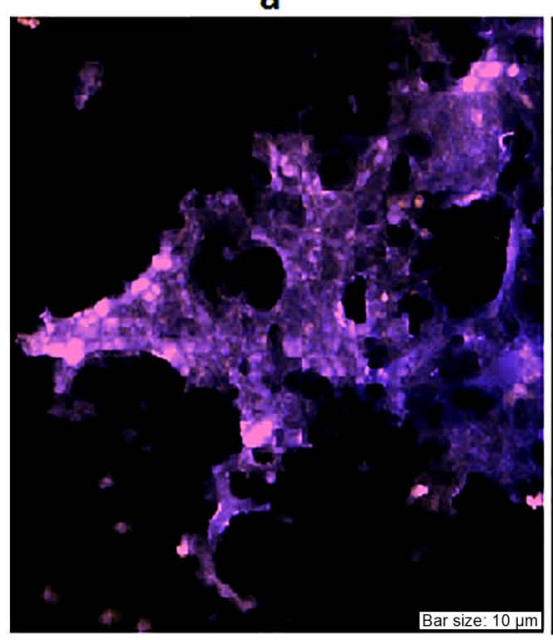

b

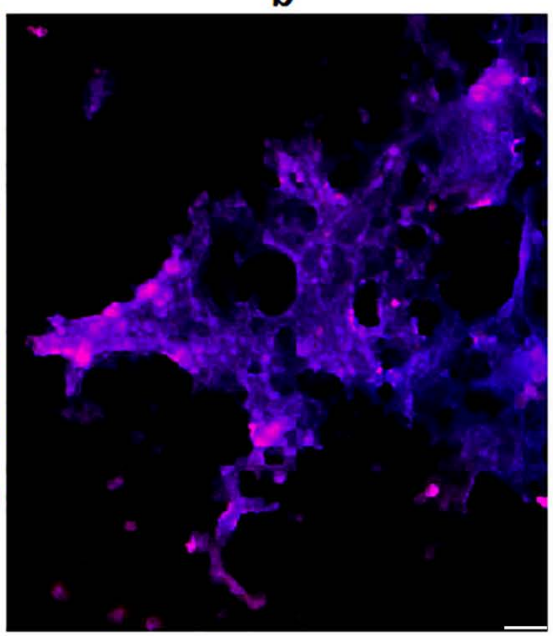

C

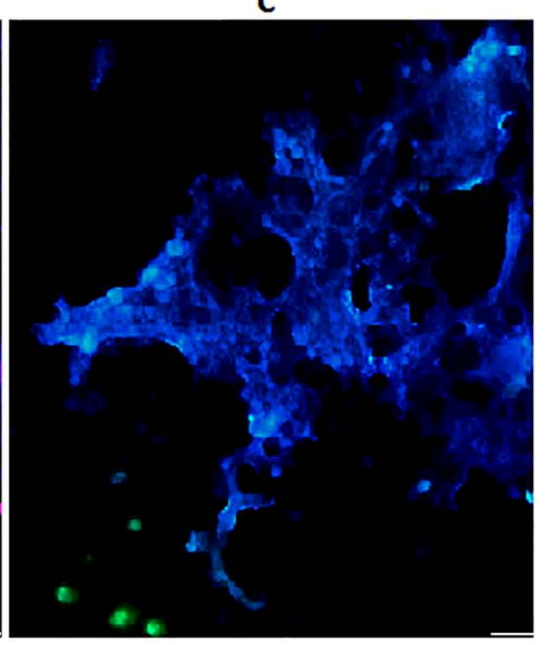

Figure 1: Mode of protein expression for VEGF, EGF, and CCL2 in section 1 of cerebellar Medulloblastoma cells.

PE: protein expression; cerebellar Medulloblastoma cells are merged with: a) Dapi/VEGF; b) Dapi/EGF; and c) Dapi/CCL2 presenting diverse expression pattern including high, intermediate, low, and cooperative manner. 


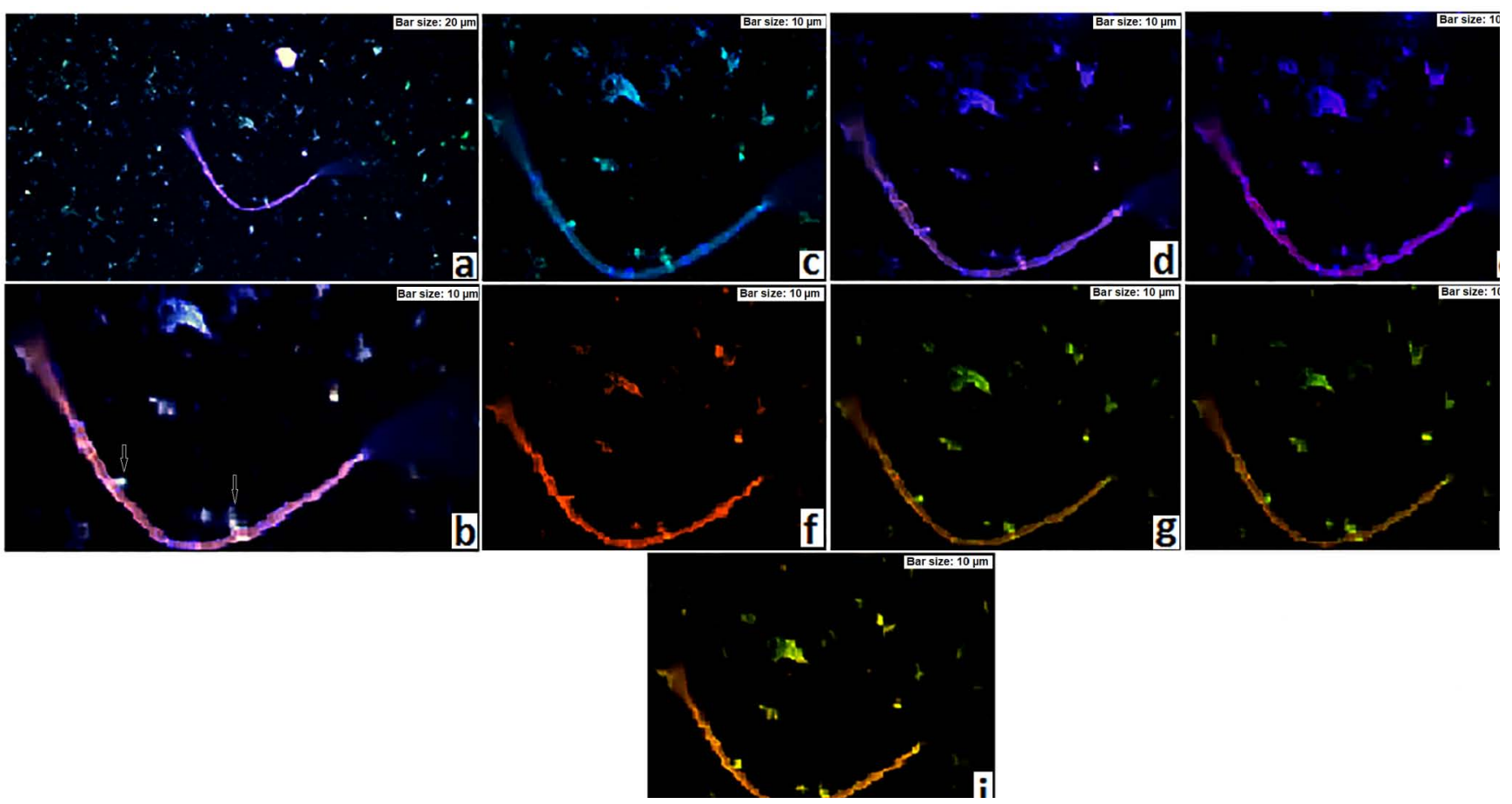

Figure 2: Status of protein expression of CCL2, VEGF, EGF in the cerebellar meduloblastoma migrated cells to the section 1 of peritoneal cavity.

Merged migrated cerebellar Medulloblastoma cells to peritoneal cavity with: a) and b) Dapi/CCL2/VEGF/EGF; c) Dapi/CCL2; d) Dapi/VEGF; e) Dapi/EGF; f) VEGF/EGF; g) CCL2/NEGF; h) CCL2/EGF and i) CCL2/VEGF/EGF. Images b, f-h indicate remarkable co-expression.

1
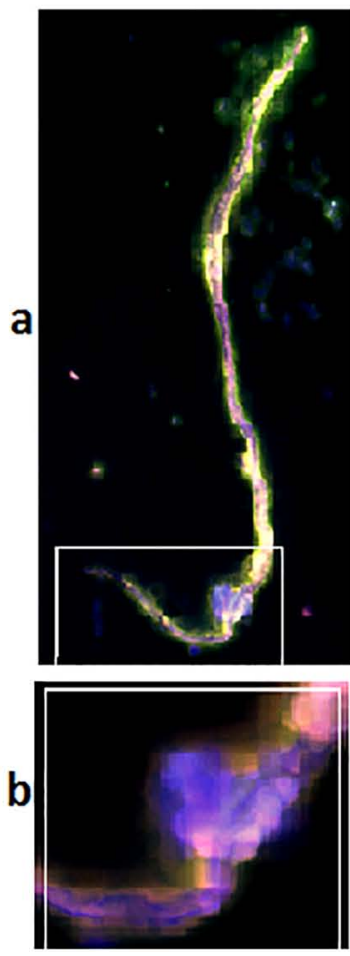

2
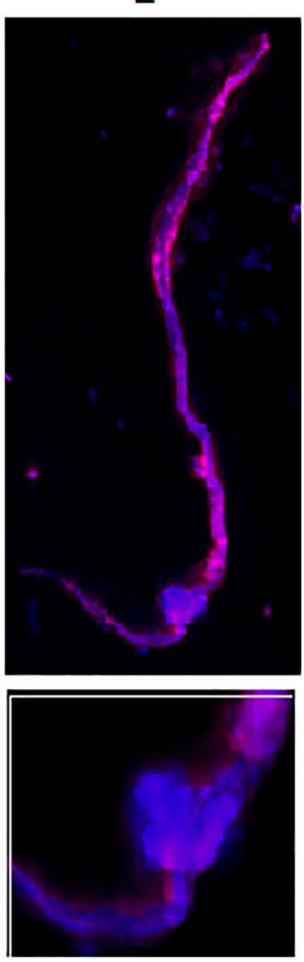

3
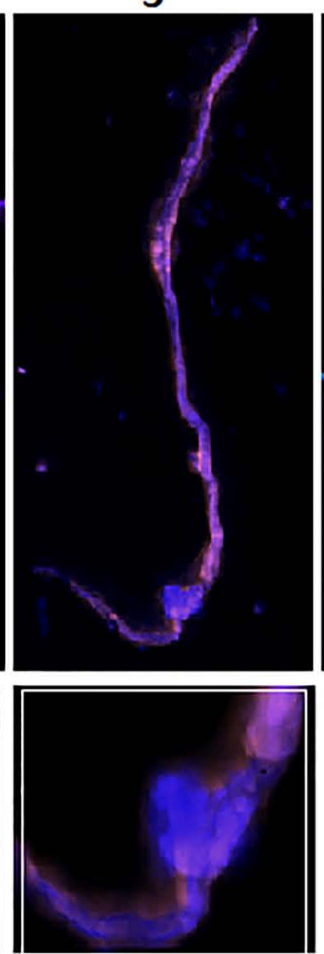

4
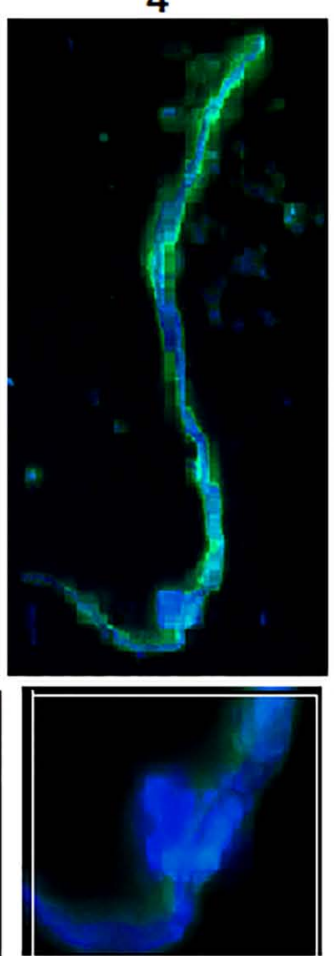

5
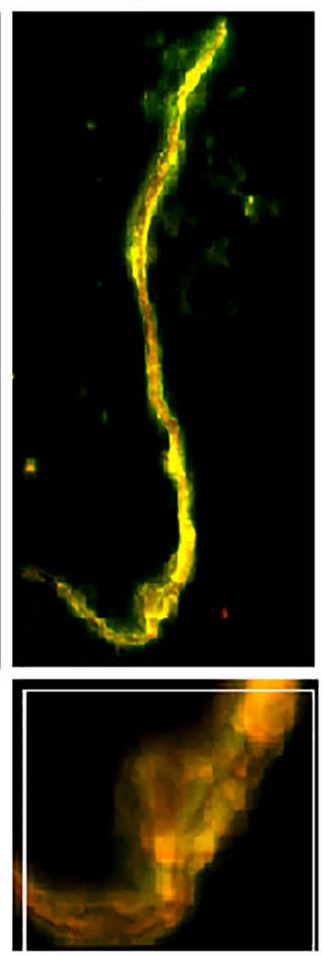

Figure 3: Status of protein expression of CCL2, VEGF, EGF in the cerebellar Medulloblastoma migrated cells to the vasculature of section 2 in peritoneal cavity.

a) Merged image with: 1) dapi/CCL2/VEGF/EGF; 2Dapi/EGF); 3) Dapi/VEGF; 4) Dapi/CCL2; 5) CCL2/VEGF/EGF. b) Cropped section of a1-5 images. Diverse protein expression (2-4) and harmonic co-expression $(1,5)$ are remarkable. 


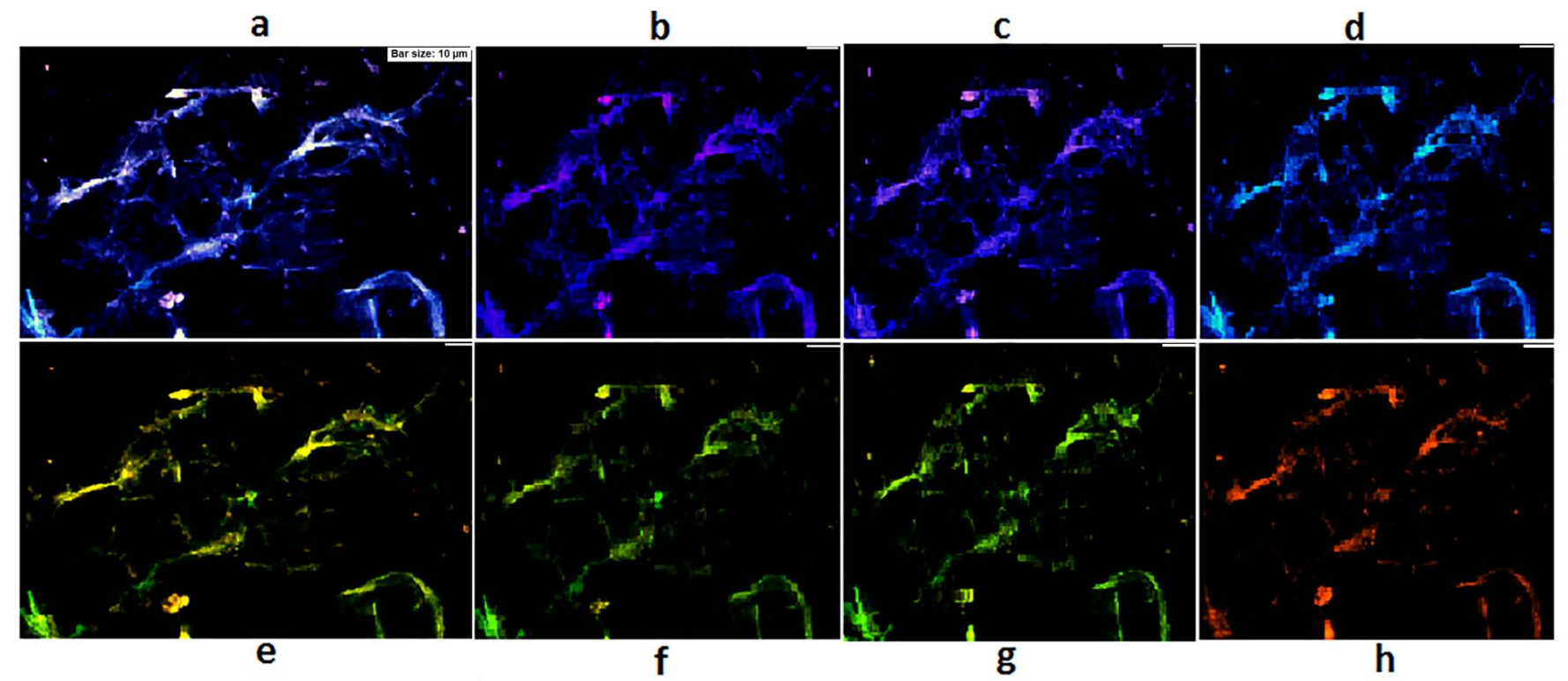

Figure 4: Mode of protein expression and co-expression of VEGF, EGF, CCL2 in the metastatic cerebellar Medulloblastoma cells to the section 3 of peritoneal cavity.

CCL2, VEGF and EGF are conjugated with FITC, R-pe and Pe-cy5 respectively. Merged of: a) Dapi/ CCL2/VEGF/EGF; b) Dapi/EGF; c) Dapi/VEGF; d) Dapi/CCL2. Co-expression of: e) CCL2/NEGF/EGF; f) CCL2/EGF; g) CCL2/VEGF; h) EGF/VEGF.

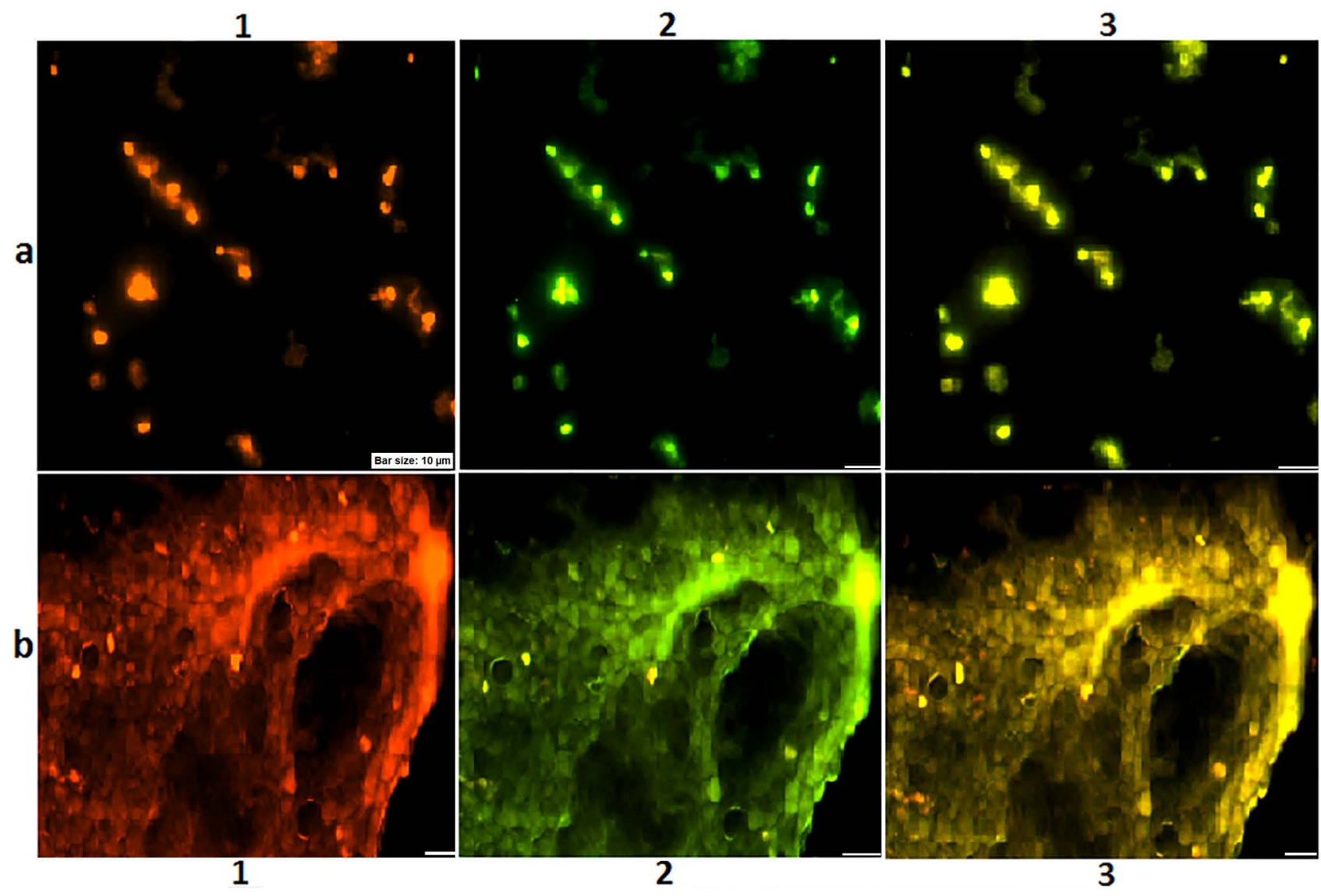

Figure 5: Co-expression of CD133, Cyclin E and P21 in the cerebellar Medulloblastoma cells of the brain and peritoneal cavity of a metastatic patient.

CD133, Cyclin E and P21 are conjugated with Rpe, FITC and Pe-cy5 respectively.

a and b) Co-expression of: 1) CD133/P21; 2) P21/cyclin E; 3) CD133/P21/cyclin E in brain region (section a) and peritoneal cavity (section b) respectively, indicating the remarkable co-expression. 

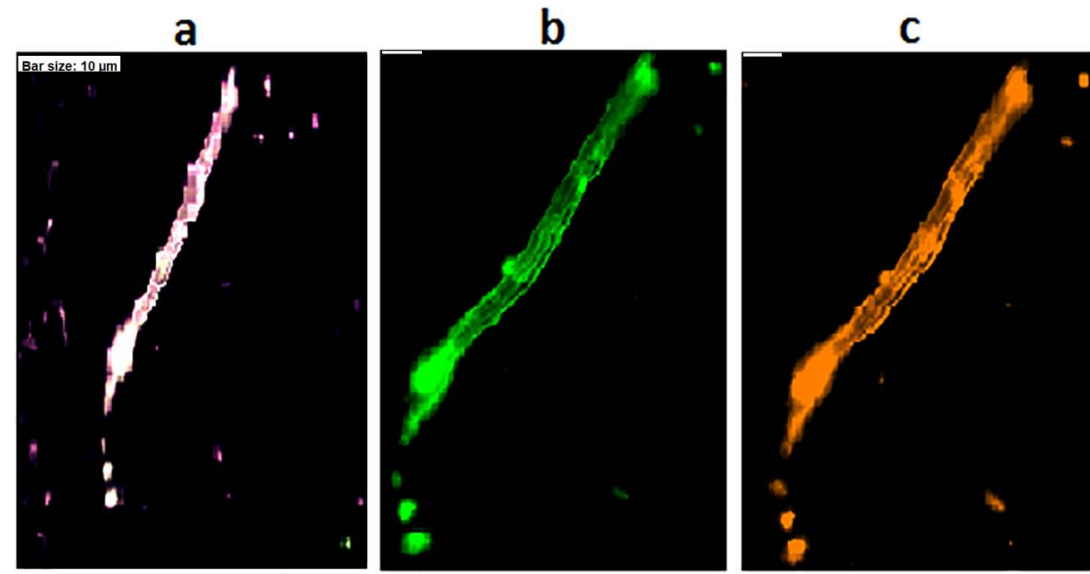

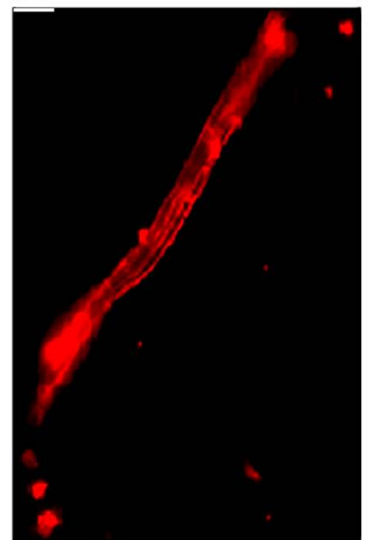

d

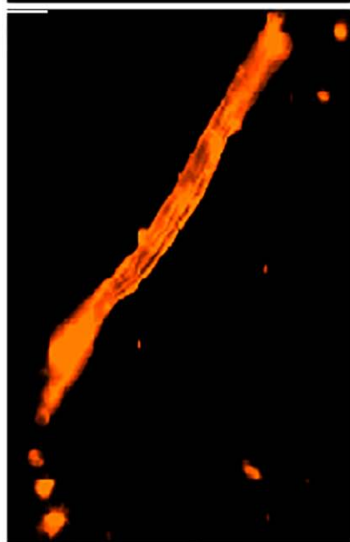

$\mathbf{e}$

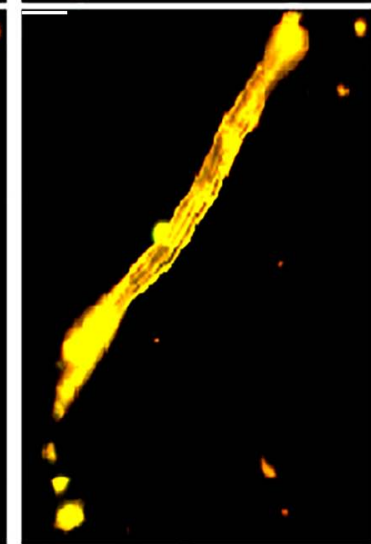

f

Figure 6: Protein expression status of neurofilament marker, CD133 and VEGF in the cerebellar meduloblastoma cells and the migrated cell to the vascular region of a metastatic peritoneal cavity with CM origin.

Neurofilament marker (NM), CD133, and VEGF are conjugated with FITC, Rpe, and Pe-cy5 respectively; PC: peritoneal cavity; CM: cerebellar meduloblastoma. Merged cerebellar meduloblastoma cells of: a) Dapi/NM/CD133/VEGF; b) neurofilament marker; c) CD133; d) VGF; e) D133/VEGF, f) NM/CD133/VEGF. Harmonic expression and co-expression in neurofilament marker, CD133 and VEGF is noticeable. Dual (e) and triangle (f) co-expression is remarkable.

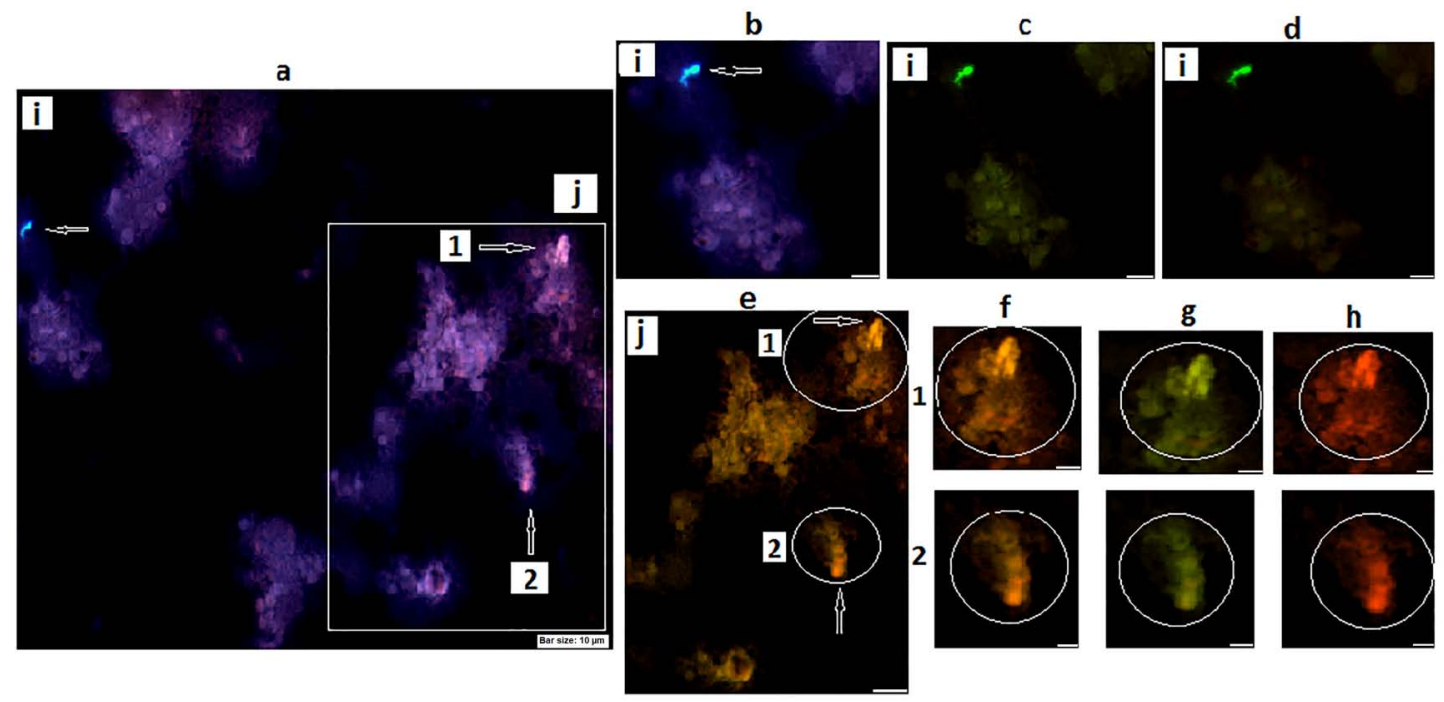

Figure 7: Status of protein expression and co-expression of brain CTCs in a patient with metastatic peritoneal cavity with cerebellar Medulloblastoma origin.

CCL2, VEGF and EGF are conjugated with FITC, R-pe and Pe-cy5 respectively.

CTCs images with merged of: a) Dapi/CCL2/VEGF/EGF; b: partial image of a-i; c-i) CCL2/VEGF; d-i) CCL2/EGF; e) Cropped image of a-j1 \& a-j2 (e1 \& e2) respectively: CCL2/VEGF/EGF; f-h (1 \& 2: cropped from e): CCL2/VEGF/EGF; CCL2/VEGF and VEGF/EGF respectively. 


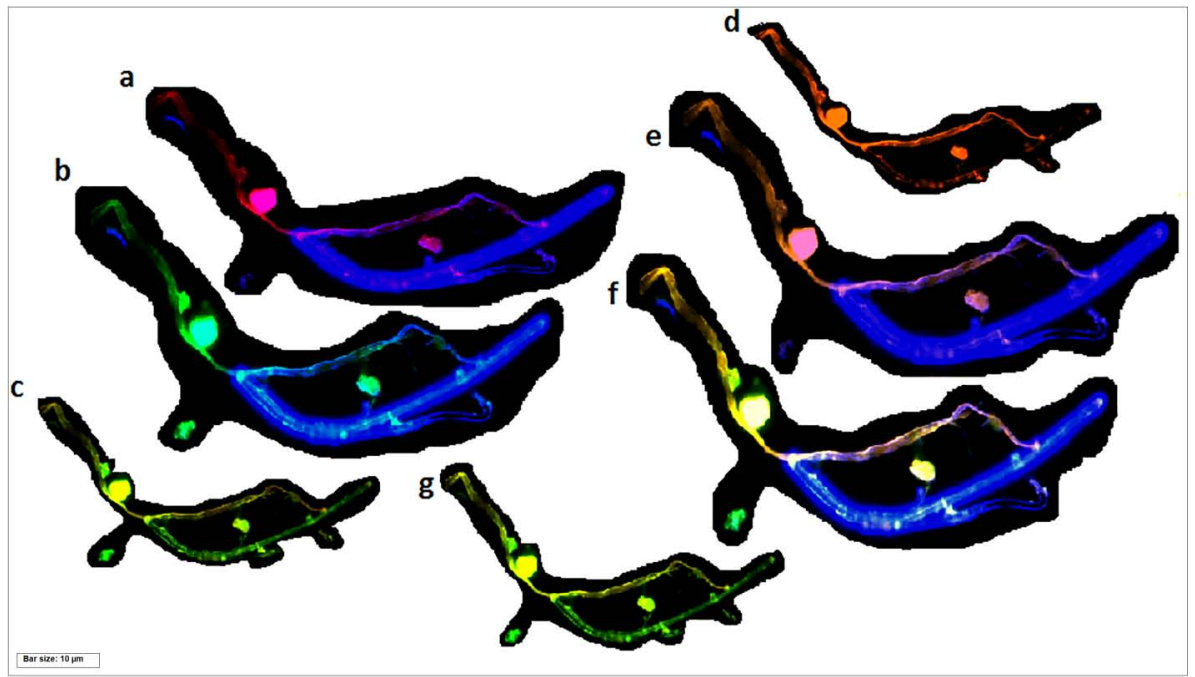

Figure 8: Mode of protein expression of CD133, Cyclin E, and P21 in CTCs of a patient with metastatic peritoneal cavity with cerebellar Medulloblastoma origin.

Cyclin E; CD133 and P21 conjugated with FITC, Rpe and Pe-cy5 respectively. Images merged with: a) Dapi/P21with lowest cell frequency with P21; b) Dapi/Cyclin E; c) Cyclin E/P21; d) CD133/P21; e) Dapi/CD133; f) Dapi/ Cyclin E/CD133/P21; and g) Cyclin E/Cd133. Dual or triangle harmonic co-expression is remarkable between these proteins. (adopted from Mehdipour's archive]).

a
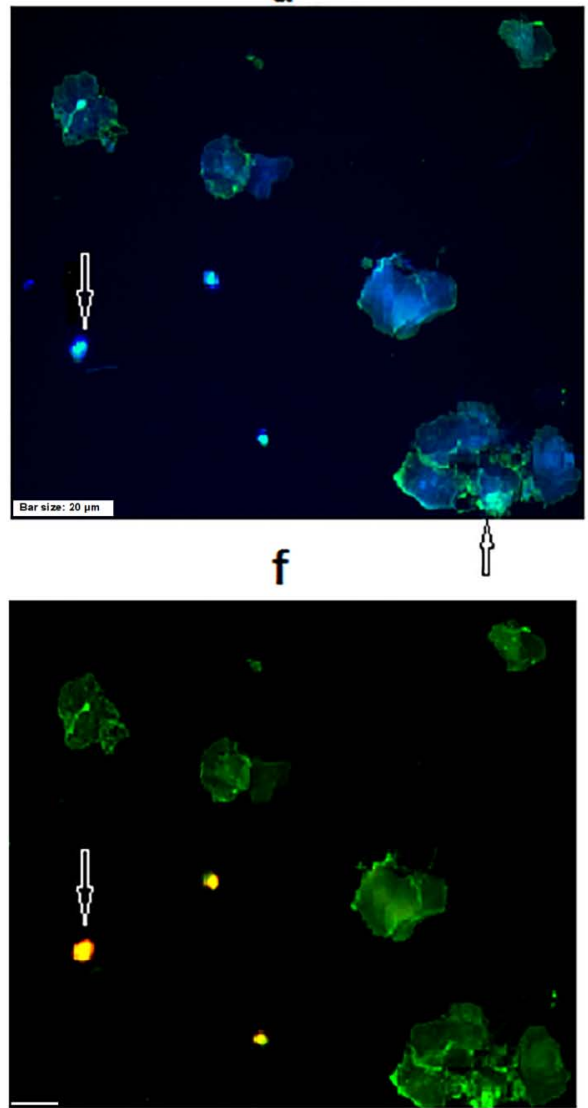

b

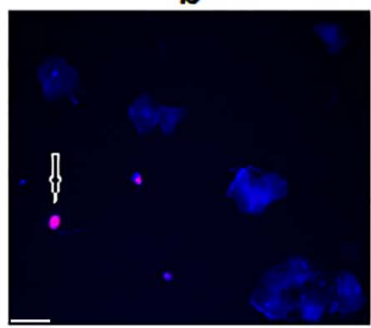

d

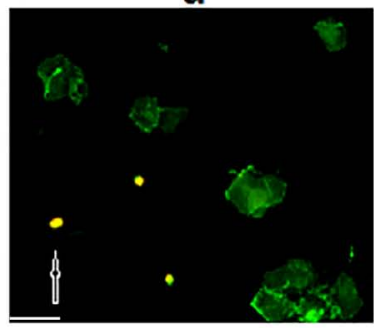

g

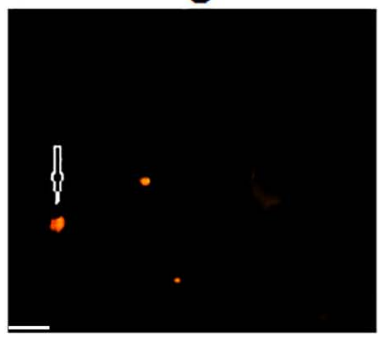

C

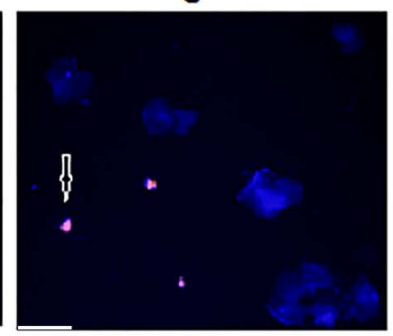

e

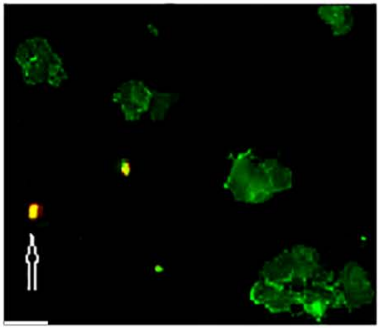

h

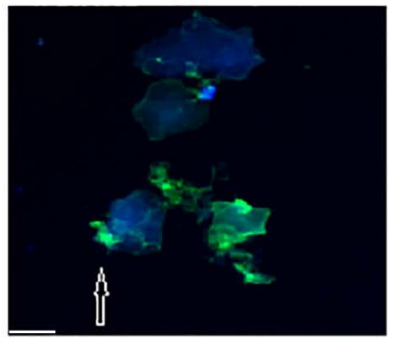

Figure 9: Protein expression and co-expression of CCL2, VEGF and EGF of migrated cells to the buccal region.

CCL2, VEGF and EGF are conjugated with FITC, R-pe and Pe-cy5 respectively. The $\mathrm{h}$ reflects another region of buccal cells with merged of dapi/NM/CD45.

Images merged with: a) Dapi/CCL2; b) Dapi/EGF; c) Dapi/VEGF; d) CCL2/VEGF; e) CCL2/EGF; f) CCL2/EGF/VEGF; and g) EGF/NEGF. The $\mathbf{h}$ ) reflects merged of Dapi/NM from a complementary buccal cells conjugated with NM+/CD45-. 
respectively presented (Figures 7 and 8). As a complementary strategy, PE and co-expression of CCL2, VEGF and EGF of migrated cells to the buccal region is also provided (Figure 9). Finally, the pulse system, based on T/CTCs-ratio, is created as a predictive applicable graph for: 1) the primary CMtumor and 2) host organ (PC) for clinical managements (Figure S2 and S3).

\section{DISCUSSION}

Metastatic event is a major challenging paradigm in cancer- research and clinic. The notable concern is related to the nature of fundamental events to initiate and progress the metastatic events. Metastasis is a programming cascade in which the heterogenic pattern of cellular functions plays the most influential role; it not only relies on the predominance manner of cell clones, but also on the cooperation between different proteins as the final truncated production of the molecular alteration (s). Extracranial migration through ventricleperitoneal shunt could mostly occur at intracranial surgery [10]. However, our results does not support the occurrence of such event as a sole factor, rather it reflects a programmed scenario and predisposition in favor of metastatic event to the peritoneal destination. Absence of PE in tumor cells (TCs) and CTCs is a fact through the cell migration including metastasis (Figure S1). The diverse ratios of TCs/CTCs, remarkably, reflect higher value in TCs, either for T-cells (sections $1-4$ ) in the brain (Figure S1a), or in the migratory cells (sections 1-3) in PC (Figure S1b), and the highest value was observed for EGF. Regarding the cells with positive $\mathrm{PE}$, the diverse ratios are observable in a and b categories for intensities, including C1-3, V1-3 and E1-3 (Figures S1a and S1b), with remarkably lower value than $\mathrm{C} 0, \mathrm{~V} 0$, and $\mathrm{E} 0$. These findings indicate that more cells with different intensity of PE have migrated from $\mathrm{CM}$ to the PC. However, the harmonic PE between different intensities, and the ratio less than 1 for $\mathrm{C} 1$, as a poor prognosis, may have the applicable impacts on the clinical managements.

\section{PROTEIN EXPRESSION MODE IN PRIMARY AND HOST REGIONS}

More CM cells with high PE are observed in VEGF than in EGF, but not for CCL2 (Figures 1a, 1b, 1c), instead the released cells had high PE (Figure 1c) which reflects the occurrence of a functional evolution through the process of migration. This manner of PE is the supportive data regarding the "seed and soil" theory. The CTCs (seeds) in the vasculature region have arrived in the $\mathrm{PC}$ region and may migrate to the second destination in future. At a glance, the mode of PE is diverse between CTCs and the free cells in PC (Figure 2a-e). So, the precise classifications of the PE-behavior will unmask the metastatic strategy. Diverse PE and remarkable co-expression reflect the cellular heterogeneity (Figure $\mathbf{2 f - i}$ ).

The bulk of $\mathrm{CM}$ cells has migrated from brain to vasculature system, and then to $\mathrm{PC}$ where colonies of cells may have been gradually formed in the host (Figures 3a, 3b-1-3). Such mechanism would guarantee the metastatic process. In some region of omentum mass, the vessels have high expression of VEGF, but some free cells lacking expression. Diverse $\mathrm{PE}$ and harmonic co-expression are remarkable which may be involved in progressive process of metastasis (Figures 3: a2-4 and b1-5). Regarding the metastatic $\mathrm{CM}$ cells migrated to the section 3 of $\mathrm{PC}$, the highest $P E$ is observed for CCL2, and successful dual coexpression has occurred between CCL2/VEGF and EGF/VEGF with vesicles and angiogenic elements (Figure 4d-h). In spite of diverse PE, the triangle coexpression in these proteins has occurred (Figure 4e).

The comparison of single cells with high PE between CM-cells in brain and in PC revealed the presence of more cells with successful co-expression either for CD133/P21, or P21/cyclin E, or CD133/P21/ Cycln E. Besides, more cellular diversity than in brain territory may be considered as a poor prognosis (Figures 5a and 5b1-3).

Furthermore, the triangle PE profile reflects the harmonic $P E$ and co-expression between CD133/VEGF for the migrated and free cells in the brain- and vasculature- region (Figure 6a-f). However, CD133 play the key initiative role in tumor heterogeneity/progression/evolution and the metastatic process in the CM tumor through a journey in the blood stream. Interestingly, the CTCs are characterized with a single cell with high CCL2 expression (Figure 7a, bd), this finding highlights that 1) CCL2 could independently act without the presence of EGF and VEG; and 2) this cell may be non-invasive. But, lack of expression of CCL2 and presence of high PE in VEGF and EGF are observable between merged images including Dapi/CCL2/VEGF/EGF, CCL2/VEGF/EGF, CCL2/VEGF, CCL2/EGF, and VEGF/EGF respectively (Figures $\mathbf{7 a}, \mathbf{b}$ and $\mathbf{e}-\mathbf{h}$ ). Conclusively, the migratory role of CCL2/VEGF/EGF, either as a sole or cooperative manner, led to a successful metastatic process of CM cells to the PC. 
Colonization of CTCs in the blood territory is the most remarkable in the cells with $P E$ for cyclin $E$ (Figures $\mathbf{8 b}, \mathbf{c}, \mathbf{f}, \mathbf{g}$ ) which directly reflects the expression mode of specific proteins within the migrated vasculature. It seems that cyclin E plays a directive role, followed by CD133 and P21 in tumor progression (Figures $\mathbf{8 b}, \mathbf{c}, \mathbf{e}-\mathbf{g}$ ). In addition, P21 have the security role against $C D 133$ through its harmonic high $P E$ (Figures 8a,d). The PE and co-expression between CCL2/VEGF/ EGF of the migrated cells to buccal region, demonstrates couples of single $\mathrm{CM}$ cells as CTCs conjugated with proteins C, V,E which guaranteed the migration from brain to the buccal region (Figures 9a-c). Besides, dual and triangle coexpression between these proteins indicates a cooperative manner for probable metastatic model to another tissue (Figures 9.tif: $\mathbf{f}-\mathbf{g}$ ). The tissue origin of brain cells is also confirmed by $\mathrm{NM}+/ \mathrm{CD} 45$ - (Figure 9h). So, buccal cells are a reliable candidate for exploration of initial migratory/metastatic process at every stage of cancer initiation and/or progression either in cancer patients, or in the family members who are at risk of being affected with neoplastic disorder. Furthermore, by considering the CTCs as a positive control, then comparison between T-cells in brain and $P C$, provides the informative diverse ratios. Besides, the general pattern of ratios including $>1$ and $<1$, some key differences are notable including: 1) Quantitativebased of frequency for T-cells and CTCs in two target samples; 2) Remarkable differences for the evolutionary pattern from $\mathrm{V} 0$, to $\mathrm{V} 1, \mathrm{~V} 2$ and $\mathrm{V} 3$ between CM and PC (Figure not shown Figures $\mathbf{S 2}$ and S3a,b); and 3) Distinctive poor prognosis, i.e., an index with less than 1 , is related to the ratio of T/CTCs for PC sample. Interestingly, less than 200 extracranial metastatic GBM has been reported. The second rare report is presented as the metastasis to oral/ buccal cavity [11]. Besides, the most favorable destinations of extra-cranial metastases from glioblastoma are reported to be host organs different from PC [2]. However, our patient with $\mathrm{CM}$ provides a metastatic model from brain to peritoneal cavity. Also, the local invasion and angiogenic behavior are the main characteristics of malignant brain tumors. However, the occurrence of such events has not been previously defined in the CM.

The metastatic GBM is rare, however the CTCs could be detected in more than $1 / 3^{\text {rd }}$ of GBM patients and mouse model by emphasizing on the role of CTCs on the "disease monitoring" [3]. In order to apply an influential strategy for a rapid treatment and most beneficial outcome for the extracranial metastatic patients, the cell based exploration of CCL2/VEGF/EGF profile could be a choice for the personalized management. It was referred to the survival capacity of glial tissue for imbedding in distant organ through an accidental intervention in surgery [12]. However, due to the remarkable behavior in PE of $\mathrm{C}, \mathrm{V}$ and $\mathrm{E}$ proteins, the biological requirements have facilitated the metastatic behavior in our patient with $\mathrm{CM}$. Besides, the spreading process of leptomeningeal (LM) metastasis from Medulloblastoma -CTCs to the LM- space in vivo with high CCL2 expression has also been reported [13]. Brain tumor initiating cells, known as Medulloblastoma stem cells are highlighted as a reliable model in exploring Medulloblastoma heterogeneity as well [14]. According to our data, cooperation of CD133/P21/Cyclin E reflects a precise picture of heterogeneity. Besides, positivity of D1853N as a predisposing marker is a warning prognostic factor [15].

\section{CONCLUSION}

Conclusively, present data may be considered as a personalized human model in metastatic cancers. It worth stating that the expression pattern of $C, V, E$ is diverse between different human tumors and also within the specific tumor type (unpublished data). So, by considering the key-interactive proteins, the personalized-based strategy may lead to an effective clinical management of the patients with neoplastic disorders including brain tumors. Present findings deliver the somatic/genomic ratio-based prognosis for further clinical managements. Finally, it would be helpful to predict whether the "seed \& soil" theory be repeated in this patient, and then next metastatic journey to another host is preventable.

\section{ABBREVIATIONS}

$\begin{array}{ll}\mathrm{CM} & =\text { Cerebellar Medulloblastoma } \\ \mathrm{PC} & =\text { Peritoneal Cavity } \\ \mathrm{PB} & =\text { peripheral blood } \\ \mathrm{T} & =\text { tumor } \\ \mathrm{CTCs} & =\text { Circulating tumor cells } \\ \mathrm{PE} & =\text { protein expression } \\ \mathrm{CCL}(\mathrm{C}) & =\begin{array}{l}\text { Macrophage chemoattractant C-C } \\ \end{array}\end{array}$




$$
\begin{array}{ll}
\operatorname{VEGF}(\mathrm{V}) & =\text { Vascular Endothelial Growth Factor } \\
\operatorname{EGF}(\mathrm{E}) & =\text { Epidermal growth factor } \\
\mathrm{CD} 45(\mathrm{LCA}) & =\text { leukocyte common antigen } \\
\mathrm{NM} & =\text { neuronal marker }
\end{array}
$$

\section{ACKNOWLEDGEMENTS}

The authors are indebted to the patients for participating in this study.

\section{CONFLICT OF INTEREST}

The authors have no conflict of interest.

\section{FUNDING}

Funding was supported by Tehran University of Medical Sciences, Research deputy under project No.:32208-30-04-95.

\section{CONSENT TO PARTICIPATE}

Informed consent was obtained from all individual participants included in the study.

\section{SUPPLEMENTAL FIGURES}

The supplemental figures can be downloaded from the journal website along with the article.

\section{REFERENCES}

[1] Kleihues $\mathrm{P}$, Ohgaki $\mathrm{H}$. Primary and secondary glioblastomas: from concept to clinical diagnosis. Neuro Oncol 1999; 1: 4451. https://doi.org/10.1093/neuonc/1.1.44

[2] Lun M, Lok E, Gautam S, Wu E, Wong ET. The natural history of extracranial metastasis from glioblastoma multiforme. J Neurooncol 2011; 105: 261-273. https://doi.org/10.1007/s11060-011-0575-8

[3] Sullivan JP, Nahed BV, Madden MW, Oliveira SM, Springer $\mathrm{S}$, Bhere $\mathrm{D}$, et al. Brain tumor cells in circulation are enriched for mesenchymal gene expression. Cancer Discov 2014; 4: 1299-1309.

http://doi.org/10.1158/2159-8290.cd-14-0471
[4] Kalokhe G, Grimm SA, Chandler JP, Helenowski I, Rademaker A, Raizer JJ. Metastatic glioblastoma: case presentations and a review of the literature. J Neurooncol 2012; 107: 21-27.

https://doi.org/10.1007/s11060-011-0731-1

[5] Gao F, Cui $Y$, Jiang $H$, Sui D, Wang $Y$, Jiang Z, Zhao J, Lin $S$. Circulating tumor cell is a common property of brain glioma and promotes the monitoring system. Oncotarget 2016; 7: 71330-71340. https://doi.org/10.18632/oncotarget.11114

[6] Rossi D, Zlotnik A. The biology of chemokines and their receptors. Annu Rev Immunol 2000; 18: 217-242. https://doi.org/10.1146/annurev.immunol.18.1.217

[7] Koch AE, Halloran MM, Haskell CJ, Shah MR, Polverini PJ. Angiogenesis mediated by soluble forms of E-selectin and vascular cell adhesion molecule-1. Nature 1995; 376: 517519. https://doi.org/10.1038/376517a0

Sainsbury JR, Farndon JR, Sherbet GV, Harris AL. Epidermal-growth-factor receptors and oestrogen receptors in human breast cancer. Lancet 1985; 1: 364-366.

[9] Mehdipour P, Karami F, Javan F, Mehrazin M. Linking ATM promoter methylation to cell cycle protein expression in brain tumor patients: cellular molecular triangle correlation in ATM territory. Molecular Neurobiology 2015; 52: 293-302. https//doi.org/10.1007/s12035-014-8864-9

[10] Narayan A, Jallo G, Huisman TA. Extracranial, peritoneal seeding of primary malignant brain tumors through ventriculo-peritoneal shunts in children: Case report and review of the literature. Neuroradiol J 2015; 28: 536-539. https://doi.org/10.1177/1971400915609348

[11] Kup PG, Nieder C, Winnekendonk G, Adamietz IA, Fakhrian $\mathrm{K}$. Extracranial oral cavity metastasis from glioblastoma multiforme: A case report. Mol Clin Oncol 2016; 5: 437-439. https://doi.org/10.3892/mco.2016.991

[12] Goodwin CR, Liang L, Abu-Bonsrah N, Hdeib A, Elder BD Kosztowski T, Bettegowda C, Laterra J, Burger P, Sciubba DM. Extraneural Glioblastoma Multiforme Vertebral Metastasis. World Neurosurg 2016; 89: 578-582 e573. https://doi.org/10.1016/j.wneu.2015.11.061

[13] Garzia L, Kijima N, Morrissy AS, De Antonellis P, GuerreiroStucklin A, Holgado BL, Wu X, Wang X, Parsons M, Zayne K. A hematogenous route for medulloblastoma leptomeningeal metastases. Cell 2018; 172: 1050-1062. e1014.

[14] Manoranjan B, Venugopal C, McFarlane N, Doble BW, Dunn SE, Scheinemann K, Singh SK. Medulloblastoma stem cells: modeling tumor heterogeneity. Cancer Lett 2013; 338: 23-31. https://doi.org/10.1016/j.canlet.2012.07.010

[15] Karami F, Mehdipour P. Genetic and Cellular Complexity of Brain Tumors. In :Cancer Genetics and Psychotherapy. Mehdipour $\mathrm{P}(\mathrm{ed}), 1^{\text {st }}$ edn, Springer, Cham Switzerland 2017; pp. 627-665.

\section{https://doi.org/10.30683/1927-7229.2021.10.02}

(c) 2021 Mehdipour et al.; Licensee Neoplasia Research.

This is an open access article licensed under the terms of the Creative Commons Attribution Non-Commercial License (http://creativecommons.org/licenses/by-nc/3.0/) which permits unrestricted, non-commercial use, distribution and reproduction in any medium, provided the work is properly cited. 